\title{
Le manuscrit 1 de Semur-en-Auxois
}

\section{Eduardo Henrik Aubert, Daniel Russo et Eliana Magnani}

\section{(2) OpenEdition}

\section{Journals}

\section{Édition électronique}

URL : https://journals.openedition.org/cem/11561

DOI : $10.4000 /$ cem. 11561

ISSN : 1954-3093

Éditeur

Centre d'études médiévales Saint-Germain d'Auxerre

Édition imprimée

Pagination : 101-112

ISSN : 1623-5770

\section{Référence électronique}

Eduardo Henrik Aubert, Daniel Russo et Eliana Magnani, "Le manuscrit 1 de Semur-en-Auxois », Bulletin du centre d'études médiévales d'Auxerre | BUCEMA [En ligne], 14 | 2010, mis en ligne le 14 octobre 2010, consulté le 22 septembre 2022. URL : http://journals.openedition.org/cem/11561 ; DOI : https:// doi.org/10.4000/cem. 11561

Ce document a été généré automatiquement le 22 septembre 2022.

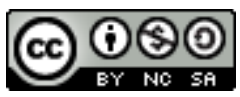

Creative Commons - Attribution - Pas d'Utilisation Commerciale - Partage dans les Mêmes Conditions 4.0 International - CC BY-NC-SA 4.0

https://creativecommons.org/licenses/by-nc-sa/4.0/ 


\title{
Le manuscrit 1 de Semur-en-Auxois
}

\author{
Eduardo Henrik Aubert, Daniel Russo et Eliana Magnani
}

$1 \mathrm{Au}$ terme des ateliers pédagogiques de recherche autour du manuscrit 1 de la bibliothèque municipale de Semur-en-Auxois, provenant de l'abbaye de Saint-Jean de Réôme/Moutiers-Saint-Jean, et en attendant la publication in extenso de cette enquête collective ${ }^{1}$, des éléments de comparaison ou de synthèse peuvent être avancés.

2 Tel qu'il nous est parvenu aujourd'hui, le manuscrit 1 de la bibliothèque municipale de Semur-en-Auxois est un volume cartonné, $260 \times 207 \mathrm{~mm}$, en parchemin, relié au $\mathrm{XIX}^{\mathrm{e}}$ siècle ${ }^{2}$, qu'Auguste Molinier (1851-1904), dans le Catalogue général des manuscrits des bibliothèques publiques de France, décrit comme «Vie de saint Jean de Réomé et mélanges sur l'histoire de Moutier-Saint-Jean». Son caractère composite et le type de pièces qu'il rassemble, font penser, effectivement, à un manuscrit, qui, au long du temps, a constitué un condensé de l'«histoire » ou de la «mémoire » de l'abbaye de Réôme, ce qui n'est sans doute pas étranger à sa conservation ${ }^{3}$. Si toutes les couches, reprises, notes et tous les ajouts ne sont pas encore entièrement éclairés, on peut aujourd'hui avancer davantage d'hypothèses pour essayer de rendre compte de leur sens.

\section{Le libellus hagio-liturgique}

3 Le libellus de Jean de Réôme (f. 0-69), des alentours de l'an Mil (?), est un ensemble hagio-liturgique à deux parties: après les leçons pour l'anniversaire, puis pour la translation du saint (BHL 4428, 4425, 5741, 4429, 4430; f. 0v-45r), marquées par des bifeuillets monumentaux à leur début (f. $0 \mathrm{v}-1 \mathrm{r}$ et $15 \mathrm{v}-16 \mathrm{r}$ ), rythmées ensuite par des lettrines savamment décorées, et les pièces liturgiques neumées (f. 47r-49r), on lit la vie du saint copiée en deux livres, qui sont annoncés aussi par des initiales décorées (f. 52v et 63v), et divisés en 16 et 12 chapitres respectivement (BHL 4426 ; f. 50r-69v). Les notes et grattages en marge indiquent les révisions des leçons de la translation (f. $20 \mathrm{v}-44 \mathrm{r}$; BHL 5741, 4429, 4430), à des périodes peut-être pas très éloignées (?) de la confection du libellus: ils pourraient témoigner, également, d'un office encore mal fixé, en cours d'élaboration ou en voie de transformation. 


\section{L'office}

4 La base de l'office semble être déjà raisonnablement ancienne au moment de la confection du libellus, comme l'attestent les pièces de chant en prose - remaniées pour la majeure partie du texte de la BHL 4428 - et l'absence d'ordre modal des répons, dont les modes peuvent être inférés par la notation, très formulaire, des versets. Mais cet office, qui peut être daté $d u \mathrm{Ix}^{\mathrm{e}}$ siècle (ou même avant), a été pendant longtemps remanié dans le double sens de la quête de complétude - détermination de toutes les pièces liturgique de l'office - et d'une croissante "properisation » - le plus grand nombre des pièces devant être exclusives des fêtes de saint Jean. La confection du libellus en est un moment fort. En effet, quelques antiennes pour l'office de l'anniversaire, dont le texte n'est pas propre à saint Jean et qui se rencontrent dans les offices d'autres saints, ont reçu des précisions dans l'écriture neumatique, qui suggèrent qu'elles n'étaient pas aussi connues que les autres au moment de la confection du libellus. Vraisemblablement, il s'agissait d'un ajout plus récent à des couches déjà plus anciennes et assimilées. L'effort s'est d'abord concentré sur l'office de l'anniversaire, qui est déjà très complet et stable dans la première campagne de préparation du libellus. Ensuite, c'est l'office de la translation qui semble avoir été l'objet d'un remaniement: d'un côté, la réorganisation des leçons met l'accent sur saint Jean et réduit l'importance de saint Maurice d'Agaune et ses compagnons, fêtés le même jour; de l'autre, on ajoute du matériel pour cet office, avec les deux répons et les deux antiennes supplémentaires entrés certainement encore au XI ${ }^{\mathrm{e}}$ siècle et selon toute vraisemblance pour l'office de la translation, une fois que tous les grands répons de l'anniversaire avaient déjà été mis par écrit par le notateur des feuillets $47 \mathrm{r}$ à 49 r.

5 Dans cette composition, articulée autour du fondateur et des premiers temps de l'abbaye, les récits des translations et des miracles posthumes de Jean (BHL 4429, 4430) inscrivent la communauté dans la durée, tandis que la passion de saint Maurice et de ses compagnons (BHL 5741), commémorée à la même date que la première translation (22 septembre), associe Jean, saint confesseur mort paisiblement à cent vingt ans, au sacrifice des martyrs d'Agaune. La proximité paléographique dans l'écriture de l'ensemble, en carolines rondes et régulières, la transcription en continu sans vraie rupture entre les cahiers, malgré le passage à partir du f. 45 de cahiers à pages de 20 lignes au lieu de 19 auparavant, tout suggère l'exécution d'un projet unitaire, et d'un seul tenant, mais qui a pu connaître plus d'une campagne.

6 Sur cette réalisation de prestige, en l'honneur du saint fondateur, peut-être décidée dans le contexte de la réforme dans la mouvance clunisienne - Maïeul, Guillaume de Volpiano et Heldric ont été successivement abbés de Réôme -, on a dû, en effet, revenir à plusieurs reprises. Des ajouts successifs en témoignent: les pièces avec neumes additionnées peu après au f. $45 \mathrm{v}$; des textes lyriques transcrits au f.50r encore au $\mathrm{XI}^{\mathrm{e}}$ siècle ou au début $\mathrm{du} \mathrm{XII}^{\mathrm{e}}$ siècle, au plus tard; des compositions versifiées en hexamètres dactyliques ${ }^{4}$ adjointes au début et à la fin des leçons de l'office de l'anniversaire (BHL 4428a et $4428 \mathrm{~b}$; f. Or et $15 \mathrm{r}$ ), probablement au XI ${ }^{\mathrm{e}}$ siècle. C'est aussi au XI ${ }^{\mathrm{e}}$ siècle que le libellus a servi de base pour la copie d'une section du manuscrit Vat. Reg. 493, sûrement écrit à ou pour Saint-Germain d'Auxerre, indice de la diffusion de l'office récemment mis par écrit et en cours de fixation. À ce moment, l'attention se concentre sur l'office de l'anniversaire : les pièces de chant pour la translation n'y sont pas notées. Probablement, la transmission s'est fait très peu de temps après le premier 
effort de réalisation du libellus. Ensuite, le travail d'élaboration liturgique s'est poursuivi: on a conservé le bi-feuillet d'un bréviaire réômais - archives départementales de la Côte-d'Or, série B II 1104, liasse 11 -, datant de la seconde moitié $\mathrm{du} \mathrm{XII}^{\mathrm{e}}$ siècle, avec l'office de l'anniversaire de saint Jean dans un stade évolué (et plus développé) par rapport au libellus.

\section{Tableaux et lettrines}

Du point de vue des grands feuillets présentés sous le format de tableaux, «à l'antique», en reprenant le module calligraphique et les principaux signes d'une exposition soignée et monumentale, comme du point de vue des lettrines figurées que contient le manuscrit, la tension est forte entre un style qui pourrait relever de ce qu'Edgar de Bruyne (1898-1959), à la suite de Louis Courajod (1841-1896) du reste, n'hésitait pas à ranger du côté du "primitivisme», et une autre manière, toute empreinte de classicisme, qui retracerait les voies et les moyens de l'assimilation du classique dans l'art carolingien ${ }^{5}$. Il devait très vite, cependant, nuancer son propos en remarquant qu'il ne fallait pas trop accentuer l'opposition entre les deux esthétiques: «(...) Lorsqu'on oppose le primitivisme et le classicisme, il faut s'entendre. En effet, il ne s'agit pas plus d'un art barbare sans mélange que d'un art classique pur ${ }^{6} . »$ À l'analyse, c'est ce qui transparait de la suite des lettrines comme des deux mises en tableau monumentales (f. 0v-1r; f. 15v-16r), comme une opposition maitrisée, dominée et réglée des deux principes d'organisation, qui, à terme, se renforcent l'un l'autre. À la façon de l'art insulaire, notamment irlandais, tout est contenu dans «le paraphe » et, pour limité que soit le nombre des figures et des motifs utilisés, tous portent à une très grande diversité du fait de la combinatoire déployée par l'enlumineur ${ }^{7}$. Nous insisterons sur les deux principes à l'œuvre en même temps, sans doute au sein d'un seul atelier, scriptorium, et situé selon toute probabilité sur place, à Saint-Jean de Réôme, bien informé aussi des derniers développements stylistiques du temps. 


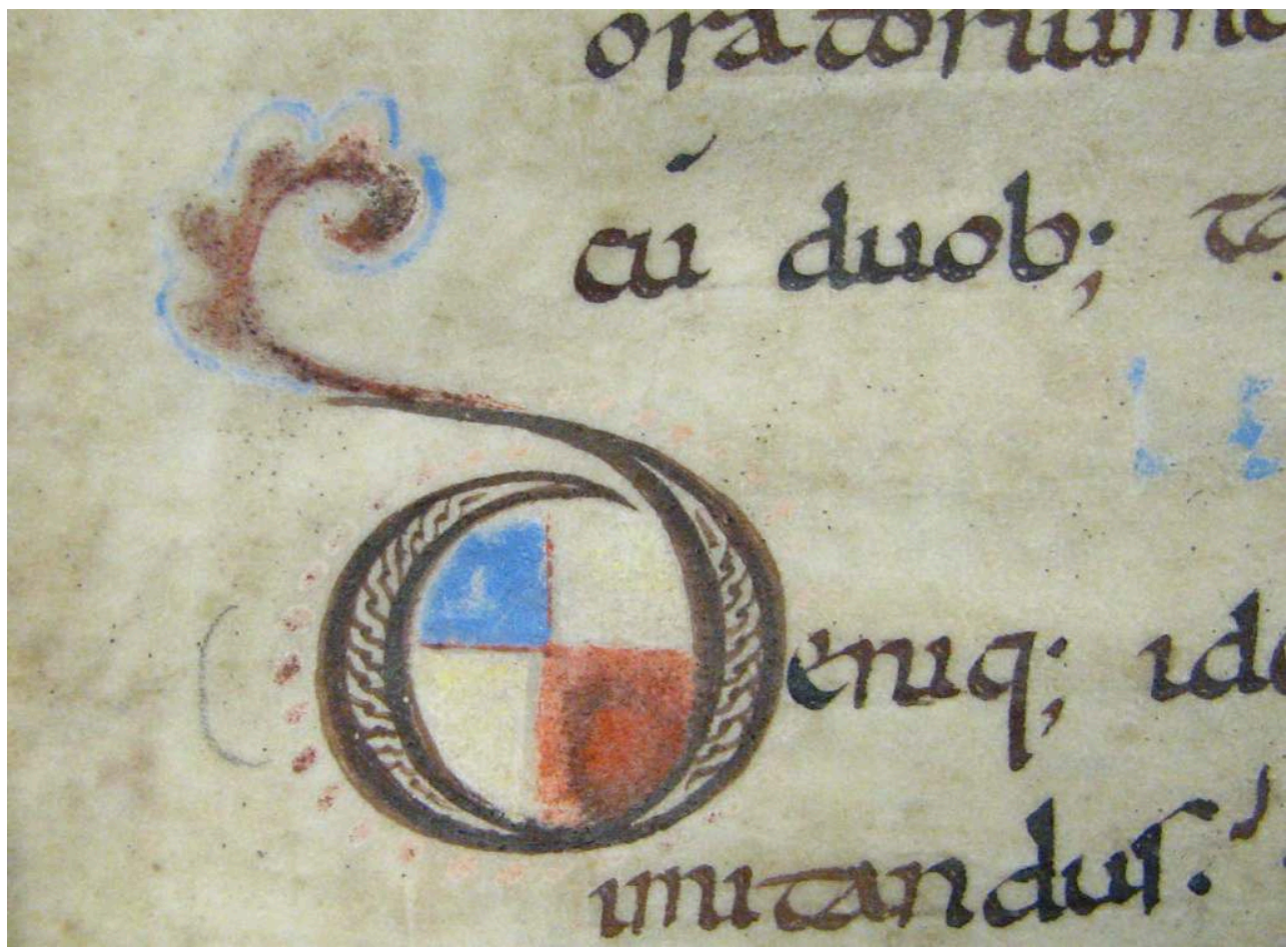

Fig. 1 - Semur-en-Auxois, BM, 1, f. 2r.

8 La synthèse carolingienne est présente, en effet, par-delà les formes de l'art insulaire au premier rang desquelles, la spirale (f. $2 \mathrm{r}$, par exemple; fig. 1); ensuite, une contrepartie anguleuse de la spirale, mais contenue en elle et composée d'une série de lignes, d'ordinaire brisées, à angles aigus, qui se poursuivent sans s'atteindre, se rapprochent sans se toucher, en combinaisons variées de labyrinthe ou de treillis à jour (f. 12r, entre autres; fig. 2); 


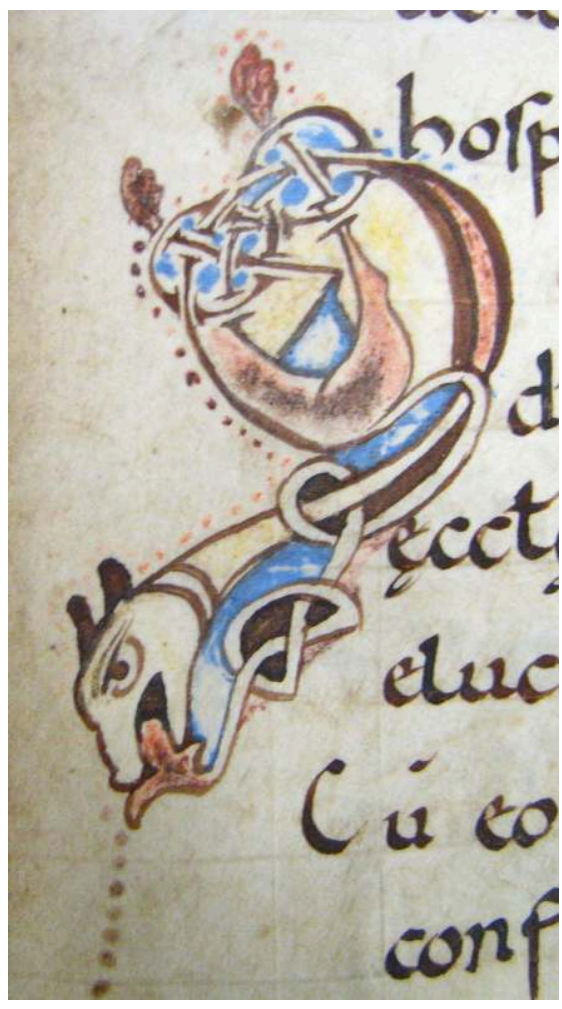

Fig. 2 - Semur-en-Auxois, BM, 1, f. 12r

9 enfin, le motif du nœud, développé dans l'entrelacs, pour former l'intérieur de la lettre (f. $24 \mathrm{v}$, f. $5 \mathrm{v}$, respectivement; fig. 3 et 4 ) -,

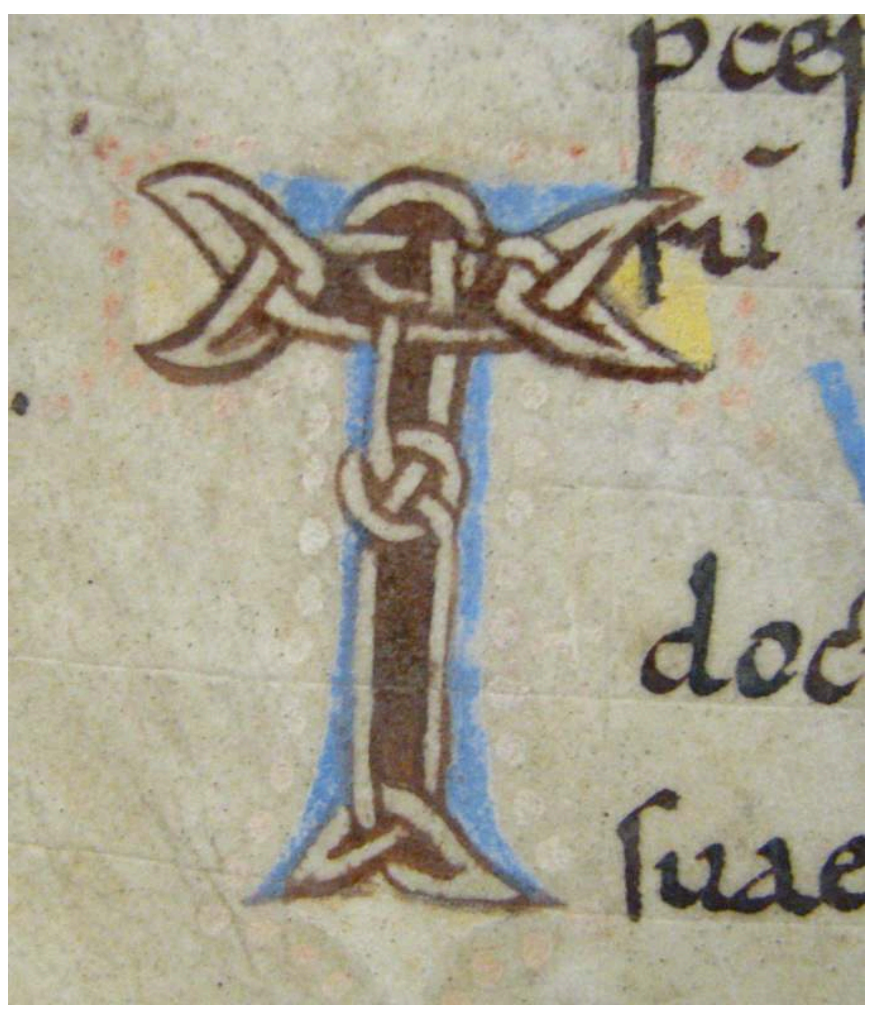

Fig. 3 - Semur-en-Auxois, BM, 1, f. 24v. 


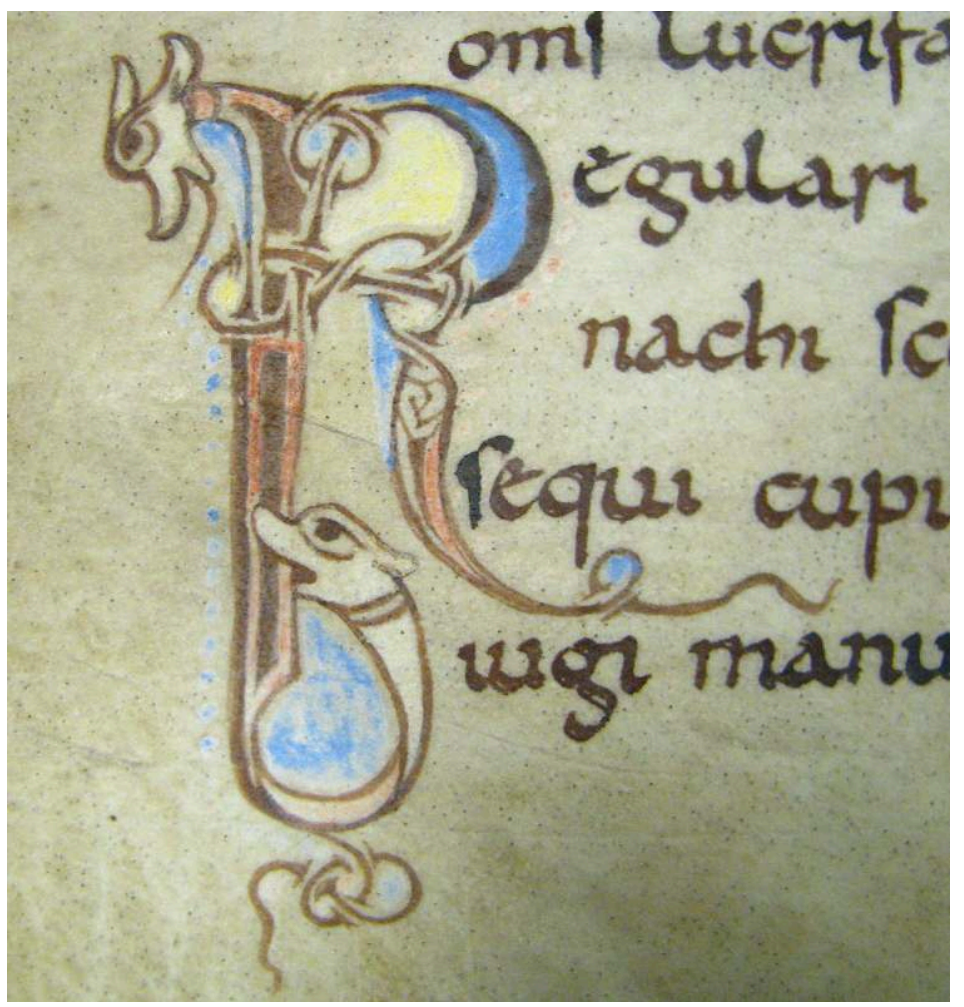

Fig. 4 - Semur-en-Auxois, BM, 1, f. 5v.

et elle s'épanouit dans la feuille d'acanthe, ordonnée en frise - f. 0v, f. 15v, à l'intérieur des bandeaux formant le cadre - et orientée de manière souple, ou encore dans l'emploi constant des formes d'animaux pour décorer ou composer les lettres (f. 20r; fig. 5). Dans les ateliers de copistes, il est vrai, que les moines bénédictins en ont établi un alphabet, pourvu des noms appropriés, précisé les catégories d'usages, les genres (ichtyomorphiques, ornithoéides...) et relevé les propriétés de chacun d'entre eux, à des fins de compréhension et d'étude générale. À partir du feuillet $5 \mathrm{v}$ et jusqu'au feuillet $22 \mathrm{v}$ (fig.6), c'est l'animal tout entier, oiseau, dragon, quadrupède, dont le corps générique dessine, seul ou uni à d'autres, les contours de la lettre initiale, qui s'anime et se colore. De même, par expansion de la figuration, cette vie interne propre aux formes gagne et contamine les lettres écrites au début de chaque nouvelle "leçon » dans les parties réservées à l'anniversaire de la vie du saint et à sa translation, et au point de passage marqué, souligné ainsi, dans le corps du texte: les lettres sont colorées, pour être davantage visualisées et devenir elles-mêmes des signes au regard d'autres - par exemple, aux f. 20r, 22v ; fig. 5 et 6. 


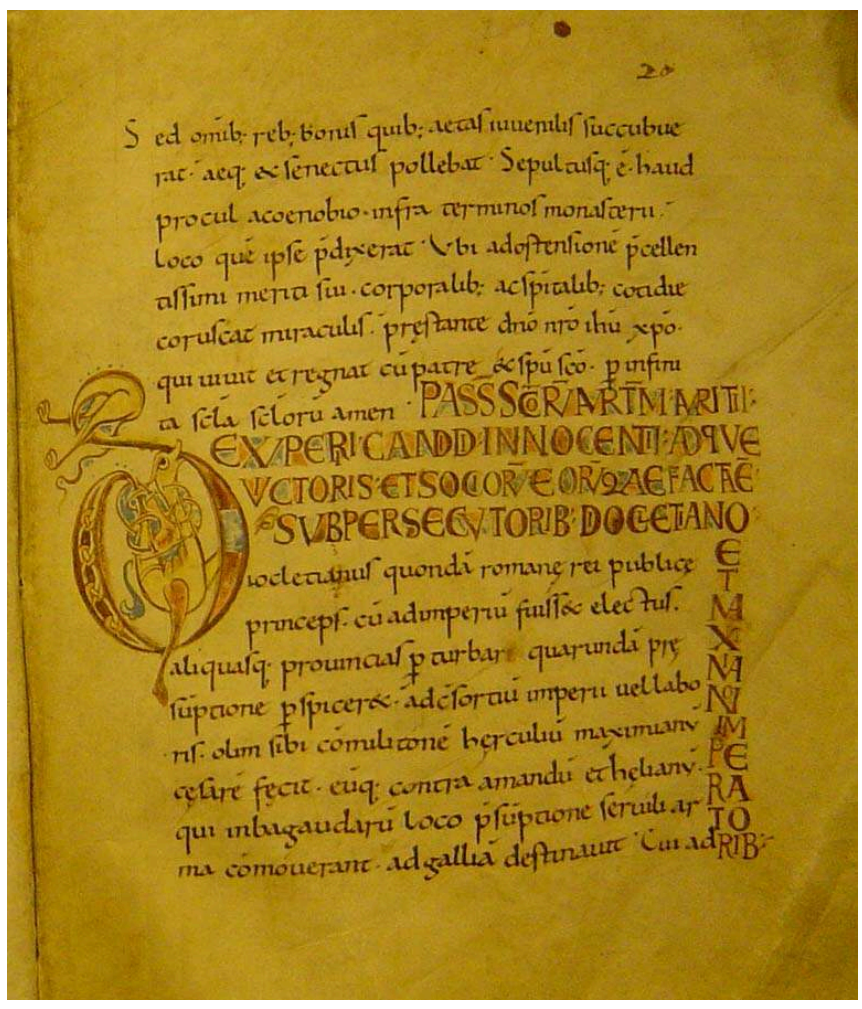

Fig. 5 - Semur-en-Auxois, BM, 1, f. 20r.

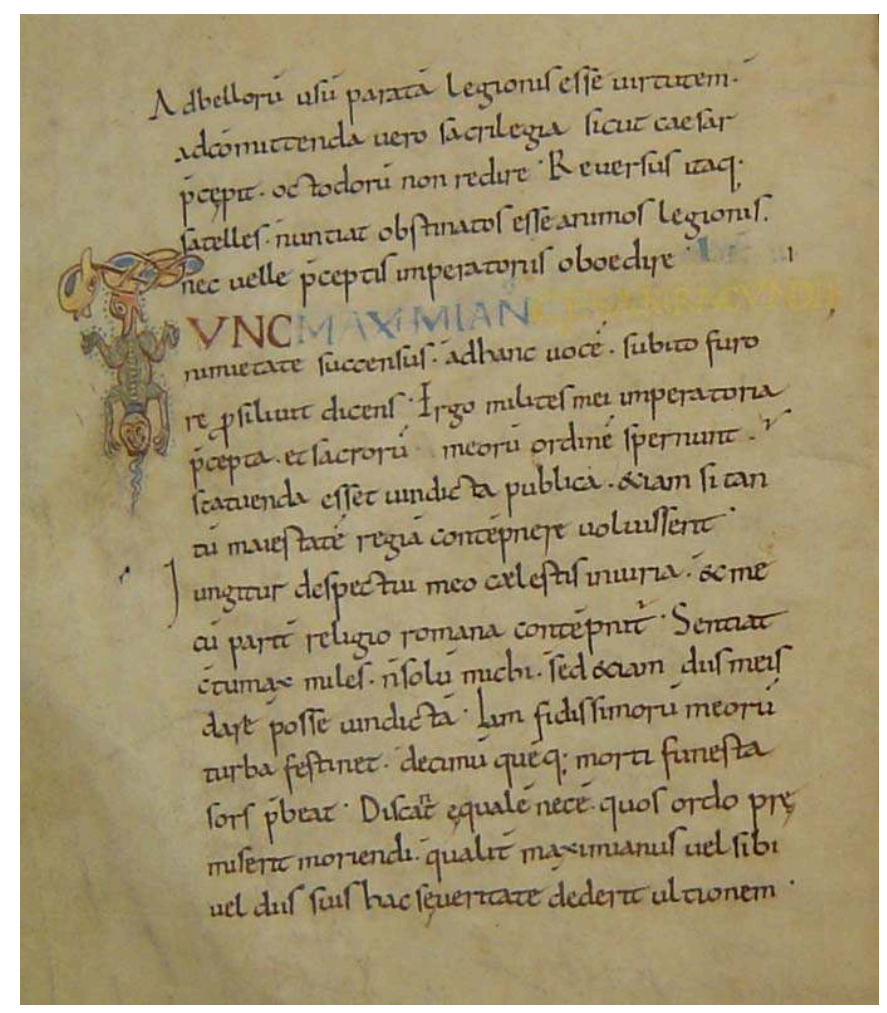

Fig. 6 - Semur-en-Auxois, BM, 1, f. 22v.

11 La pratique retrouve celle, antérieure, des scribes de l'époque carolingienne, à Corbie, au VIII siècle, à Saint-Riquier, à la fin du siècle, ou encore dans l'école du Palais, au temps de Charles le Chauve, dans la seconde moitié du IXe siècle, à l'intérieur de 
protocoles et de formules empreints de classicisme, pour ces deux derniers exemples ${ }^{8}$. Nous notons que la synthèse figurative est réactualisée autour de l'an Mil, à Saint-Jean de Réôme, et ailleurs aussi, toujours dans le sens de l'équilibre classique. Nous l'observons pareillement dans la sculpture des chapiteaux qui restent de l'époque, et qui proviennent de la région, en particulier de l'ancienne abbaye, tel le chapiteau aujourd'hui conservé dans la collection Peyre ${ }^{9}$. Sur la base de la mise en série, ponctuelle, des figurines peintes sur les feuillets du manuscrit et des corbeilles sculptées des chapiteaux, nous pensons que nous avons affaire au même mouvement de réactualisation des formes et des motifs plus anciens, par le biais de la liturgie et de l'histoire de la communauté, qui équivaut à la reformulation, menée dans les textes comme dans la pierre et dans l'enluminure, de l'ensemble des signes d'appartenance et d'identité propres au groupe monastique. Partout, à travers l'usage simultané de l'espace, de l'écrit et de l'image, en dehors des lieux autrefois réservés strictement à ces rituels, nous observons alors la même somme d'efforts visant à manifester d'autres médiations et d'autres supports d'intercession en direction de l'au-delà.

\section{Les diplômes royaux}

12 Nous ne saurions pas dire, avec certitude, quand, et comment, ils furent réunis au libellus, mais les diplômes supposés de Clovis $\mathrm{I}^{\mathrm{er}}$ (498) et de Clotaire $\mathrm{I}^{\mathrm{er}}(516)-\mathrm{f}$. $71 \mathrm{v}-72 \mathrm{v}$ et $\mathrm{f} .73 \mathrm{r}$-, adressés à Jean et à son successeur l'abbé Silvestre, seraient des créations contemporaines, dans le même contexte réformateur, probablement sous l'abbé Heldric (1003-1010), instigateur aussi de la fabrication d'actes royaux, carolingiens, à Flavigny et à Saint-Germain d'Auxerre qu'il dirigeait en même temps que Réôme. Comme le manuscrit hagio-liturgique de saint Jean, ces diplômes se rapportent aux débuts de l'abbaye et viennent renforcer la geste des origines. Plusieurs éléments semblent indiquer l'intertextualité tissée, avec soin, entre l'hagiographie et les diplômes: mention de Clovis et des chartes des rois francs conservées dans les archives du monastère; mention du récit de la succession de Jean par Silvestre, constituant de la sorte pour l'abbaye, comme une "généalogie» double et croisée, royale et abbatiale (Clovis/Jean, puis Clotaire/Silvestre), de père en fils (Clovis/Clotaire), de maître à disciple (Jean/Silvestre).

\section{L'atlante, les listes}

$13 \mathrm{Au}$ XIII ${ }^{\mathrm{e}}$ siècle le libellus est à nouveau visité. De cette époque daterait l'ajout d'un atlante au bas de l'initiale «R» du f. 63v (fig. 7) qu'on pourrait mettre en rapport avec les chantiers monumentaux contemporains. En effet, dans la seconde moitié du xIII ${ }^{\mathrm{e}}$ siècle, l'église abbatiale est reprise et agrandie dans son parti d'ensemble, comme dans les décors plastiques sculptés, et sans doute peints. Cette reprise de chantier est concomitante du déroulement des travaux sur le site de la nouvelle collégiale NotreDame de Semur-en-Auxois, selon une veine d'inspiration gothique et régionale, montrant la diffusion de ce style «moderne ", à l'époque, dans tout le sud de l'Auxois, puis en direction du nord. Le personnage figuré de l'atlante pourrait avoir été rajouté à cette occasion, et évoquer les figures de même facture dans la sculpture du tympan de Notre-Dame de Semur, au portail nord, dit le «Portail des Bleds ${ }^{10}$. 
14 aujourd'hui le deuxième volet du manuscrit. Sur le recto du bifolio, où avait été transcrit le diplôme de Clovis et qui était alors resté en blanc, a été copiée la liste, sur deux colonnes, des trente et une églises qui doivent la «croix» (cruces), une redevance exprimée en sous et en deniers, au sacristain [f. 71r - Iste sunt ecclesie quae debent cruces sacriste Reomensis; fig. 8]. D'une autre main, dans ce qui est aujourd'hui le verso de la liste des évêques de Langres, se trouve la liste, sur deux colonnes, des officiers du monastère associés à un montant exprimé en livres (74v). Dans la première colonne et à la fin de la seconde, sont énumérés les prieurs et les supérieurs des dépendances (prior, maior) ; dans la seconde colonne, les officiers claustraux - abbé, couvent, infirmier, aumônier, grand prieur, cellérier, chantre, sacristain et hospitalier -, suivis des nutriati (?). Les deux autres listes, qui s'adjoignent à ce volet, sont écrites d'une même main ou de deux mains contemporaines -, du milieu du xIV siècle, au plus tôt: il s'agit de la liste des évêques de Langres jusqu'en 1200 environ [74r - Catalogus seu nomina episcoporum lingonensis civititatis] et des abbés de Réôme jusqu'en $1351[75 \mathrm{v}-76 \mathrm{v}-\mathrm{Hec}$ sunt nomina abbatum monasterii Sancti Johannis Reomensis] ${ }^{11}$. De plus, aux marges des listes des noms - sur deux colonnes pour les évêques et une colonne pour les abbés -, des notes donnent des précisions, en général chronologiques, sur les personnages cités. Dans la liste des évêques, plus serrée et vite remplie, ces notes ont été portées également sur le f. 73v, indice codicologique indiquant que ces listes-catalogues se trouvent déjà associées aux diplômes de Clovis et de Clotaire, et plus généralement au libellus de saint Jean. De fait, on lit des annotations marginales de la même main que celle des catalogues dans les diplômes, et dans le libellus - f. 16v ?, 31r? ? 50r, 65v, 69r, $72 \mathrm{v}$ et $73 \mathrm{v}-$, ce qui témoigne d'une initiative qui s'inscrit en prolongement du noyau des alentours de l'an Mil. Ces «suites", en forme de liste, du XIII et du XIV siècle, rendent compte de l'horizon spatial et temporel dans lequel la communauté monastique s'inscrit, ainsi que de sa structure: le diocèse de Langres et les dépendances, qui rayonnent au-delà du diocèse, mais convergent vers le pôle abbatial ; le continuum des fonctions hiérarchiques de l'évêque et de l'abbé, en passant par les officiers claustraux et prieuraux.

\section{Interventions, interprétations}

Inscrits sur la page de garde finale (f. 78), l'armoirie et le nom de Jean de Cussigny, abbé de Réôme entre 1476 et 1490 , ainsi que la date de 1453 [lan mil iiii ${ }^{c}$ cinquante et trois le jour de ... (illisible)], posent la question de l'actualisation du manuscrit au milieu du $\mathrm{XV}^{\mathrm{e}}$ siècle. Bien que Jean de Cussigny, «licencié en droit canonique», auparavant prieur de Bragny-en-Charolais, dépendance de Saint-Martin d'Autun, dont il a postulé l'abbatiat face à Jean Rolin (1462) ${ }^{12}$, soit plus connu pour avoir été fait prisonnier lors des conflits autour de la succession du duché de Bourgogne dans les années 1480, il faudrait approfondir l'enquête et chercher un rapport plutôt dans son activité liturgique. On sait qu'en 1463 il fonde, à Saint-Jean de Réôme, des prières à la Vierge pour la commémoration des défunts et, qu'à la fin de sa vie, il institue une association de prières avec Cîteaux (1490) ${ }^{13}$. Quoi qu'il en soit, au milieu du Xve siècle, le manuscrit réunissait déjà les éléments actuels - à l'exception de l'acte de 1182, inséré au cours du XIX ${ }^{e}$ siècle, f. 77.

Bulletin du centre d'études médiévales d'Auxerre | BUCEMA, 14 | 2010 
$16 \mathrm{Au} \mathrm{XVII}{ }^{\mathrm{e}}$ siècle, le manuscrit est folioté et il manquait déjà alors un feuillet dans le cahier A (entre les f. 5 et 6), deux dans le C (entre les f. 17 et 18) et la fin du cahier K (après le f. 69) ${ }^{14}$. À ce moment, on porte en marge, sur le premier feuillet, l'annotation suivante: «à la page 72 du présent livre sont les chartres de la fondation de notre abbaye par Clovis premier roy chretien, et confirmées par Clotaire son fils en 516 ", note qui en dit long sur le tropisme exercé par ces diplômes. La fascination exercée par ces chartes, dont l'authenticité a été l'objet de débats récurrents ${ }^{15}$, est telle qu'au moment de la confection du premier catalogue des manuscrits de la bibliothèque de Semur-en-Auxois, Maillard de Chambure doit préciser que le manuscrit «est mal à propos désigné sous le nom de Cartulaire de l'Abbaye de Moutiers Saint-Jean ${ }^{16}$.

Au moment de la réforme mauriste de l'abbaye, dans les années 1630, le manuscrit a été une source importante pour le premier historiographe moderne de Moutiers-SaintJean, le père jésuite Pierre Rouvier (1572-1649), premier éditeur des textes hagiographiques, diplomatiques et du catalogue abbatial du manuscrit 1 - «depuis l'ancien manuscrit du monastère " (ex codice veteri monasterii ms.)-, pièces qu'il éparpille dans la composition chronologique, linéaire, de son Reomaus (Paris, 1637) ${ }^{17}$.

En 1726, le livre est mentionné dans le catalogue sommaire de la bibliothèque de Réôme dressé par Dom Urbain Plancher et décrit, en tête de liste, comme «un ancien manuscrit sur velin de sept à huit cens ans, contenant la vie de Saint Jean de Réôme, les leçons qu'on lit en ses festes, et deux chartes, une du grand Clovis et l'autre de son fils Chlotaire en faveur du monastère ${ }^{18}$ ». Plusieurs autres livres y sont mentionnés, mais, à part le liber capituli aujourd'hui conservé aussi dans la bibliothèque municipale de Semur-en-Auxois ( $\left.{ }^{\circ} 24\right)$, ils sont tous disparus.

19 L'intérêt pour le manuscrit dans son nouveau contexte de conservation à la bibliothèque municipale de Semur-en-Auxois, après les confiscations révolutionnaires, est très visiblement motivé par son âge : Maillard de Chambure, écrivant en 1834, après une analyse de plusieurs éléments - paléographie, décoration et notation musicale -, le date $\mathrm{du} \mathrm{XI}^{\mathrm{e}}$ siècle, mais une dizaine d'années plus tard, les rédacteurs du nouveau catalogue de la bibliothèque élargissent l'analyse, notamment de l'écriture musicale, qui «donne un cachet irrécusable d'ancienneté au manuscrit», et du coup la datation se trouve reculée au $\mathrm{x}^{\mathrm{e}}$ siècle. C'est à ce titre et avec simple mention de son âge «Vie de St. Jean de Réôme, $\mathrm{x}^{\mathrm{e}}$ siècle», que le ministère de l'Instruction publique et des Beaux-arts a adressé une lettre le 10 janvier 1900 au maire de Semur pour lui demander de prêter le manuscrit pour l'exposition universelle de $1900{ }^{19}$.

Dans ce contexte, au XIX ${ }^{e}$ siècle et après reliure, la troisième garde en papier reçoit l'intitulé «Histoire de Saint Jean de Réôme écrite par le moine Jonas en 660; suivie du diplôme de Clovis de janvier 496, et de la charte de confirmation de Clotaire. Manuscrit du $\mathrm{x}^{\mathrm{e}}$ siècle». Avec les six marques de tampon de la bibliothèque de Semur, à l'encre verte - première page de garde, page de titre, f. 0r, 76v, 78r-v -, ce sont les derniers ajouts apportés à cet ensemble millénaire. 


\section{NOTES}

1. Pour les références bibliographiques des textes cités et sur le programme pédagogique de recherche, voir : D. Russo, «Étude sur le manuscrit 1 de la Bibliothèque municipale de Semur-enAuxois : enluminure de manuscrit et réforme monastique ", Bulletin du Centre d'études médiévales d'Auxerre, 10 (2006), p.129-135 [http://cem.revues.org/document368.html]; D. Russo et E. MAGNANI, «Le manuscrit 1 de la Bibliothèque municipale de Semur-en-Auxois, provenant de l'abbaye de Saint-Jean de Réôme (Moutiers-Saint-Jean) : programme pédagogique de recherche ", Bulletin du Centre d'études médiévales d'Auxerre, 12 (2008), p. 305-306 [http://cem.revues.org/ document7212.html] ; E. H.AUBERT, E. MAGNANI etD. RUSSO, "Histoire du manuscrit médiéval, transmission des textes, des chants, compositions peintes et décors ", Bulletin du centre d'études médiévales d'Auxerre, 13 (2009), p. 143-155 [http://cem.revues.org/index11055.html].

2. Entre 1834 et 1884. En fait, dans la notice de l'«Inventaire raisonné et descriptif des manuscrits de la Bibliothèque publique de la ville de Semur», réalisée en 1834 par Charles Hippolyte Maillard de Chambure (1772-1841), on signale que le « manuscrit est en mauvais état et doit être incessamment relié ", alors que lors du récolement des manuscrits, en 1884, en vue de la réalisation du Catalogue général des manuscrits des bibliothèques publiques de France (t. 7, Paris, 1887), le manuscrit a déjà sa forme actuelle, on lit dans la contre-garde « Volume de 78 feuillets plus le feuillet A en moins 70 - 11 août 1884 ». Entre 1834 et 1884, le manuscrit a perdu le folio 70 (« une garde tirée d'un vieux rituel », selon le catalogue de 1834), et une charte-partie de 1182 lui a été annexée, collée sur un talon (f. 77).

3. Plusieurs fragments de livres liturgiques du XIII ${ }^{\mathrm{e}}$ siècle, servant aujourd'hui de couverture des liasses de documents de l'abbaye de Moutiers-Saint-Jean dans les archives départementales de la Côte-d'Or, indiquent que l'abbaye possédait plusieurs livres de la période médiévale, mais qu'ils ont été volontairement démembrés ou détruits.

4. Que Lênia Marcia Mongelli soit vivement remerciée pour l'analyse de ces vers.

5. Ed. DE BRUYNE, Études d'esthétique médiévale (Bruges, 1946), rééd. Paris, 1998, t. 1, livre 2, « La civilisation carolingienne ", chap. 1 « L'humanisme carolingien », p. 165-187, 169 et 172-173.

6. Ed. DE BRUYNE, Études..., ibid., p. 169. Mais il s'agit bien pour lui, cependant, de toujours distinguer, selon l'approche de « typologie esthétique » qu'il a fait sienne, entre le « barbare » et le « classique » (p. 170 et 171).

7. Ed. DE BRUYNE, Études..., ibid., p. 172 : « (...) ils [les motifs] atteignent pourtant à un véritable effet de richesse, grâce à l'adresse incroyable et à la variété infinie des arrangements. "

8. M.-P. LAFFITTE, Ch. DENOËL et M. BESSEYRE (collab.), Trésors carolingiens. Livres manuscrits de Charlemagne à Charles le Chauve, Paris, 2007, part. : PARIS, BnF, latin 11627, f. 1v (p. 120); PARIs, BnF, latin 4950, f. $1 \mathrm{v}-2$ (cat. 25, p. 134-135) ; PARIS, BnF, latin 13159, f. $118 \mathrm{v}-119$ (cat. 26, p. 136) ; PARIS, BnF, latin 1152, f. 26v (cat. 15, p. 108, Psautier de Charles le Chauve) ; PARIS, BnF, latin 17436, f. 31v-32 (cat. 16, p. 112-113, Antiphonaire de Charles le Chauve, dit de Compiègne).

9. C. SAPIN (dir.), Les prémices de l'art roman en Bourgogne, Auxerre/Précy-sous-Thil, 1999, part. (fig. 95), p. 111 : exemple mentionné du chapiteau de Moutiers-Saint-Jean.

10. Sur tout ceci et pour la définition progressive d'un gothique "régional ", autour du « groupe de l'Auxois " d'édifices et d'ateliers, cf. D. BORLÉE, La sculpture figurée du XIII siècle en Bourgogne, thèse de doctorat, université de Bourgogne, Dijon, 1997.

11. Les talons de deux feuillets coupés portant des fragments d'écriture de la même main indiqueraient que ces listes, ou leurs annotations, pouvaient être plus longues.

12. J.-G. Bulliot, Essai historique sur l'abbaye de Saint-Martin d'Autun de l'ordre de Saint-Benoit, Autun, 1849 , t. 2 , pièces et chartes justificatives, $n^{\circ} 5$, p. 305-318. 
13. P. ROUVIER (S. J., Le P.), Reomaus, seu Historia monasterii S. Joannis Reomaensis, in tractu Lingonensi, primariae inter gallica coenobia antiquitatis, ab anno Christi 425... Paris, 1637, p. 340-343 et 349-350.

14. La foliotation des feuillets 77 et 78 a été ajoutée au XIX siècle, peut-être à l'occasion du récolement de 1884.

15. T. KÖLZER, Die Urkunden der Merowinger, t. 1, Hanovre, 2001 (MGH, Diplomata Regum francorum e stirpe merovingica I, 2001), $\mathrm{n}^{\circ}$ 3, p. 7-10; $\mathrm{n}^{\circ} 15$ p. 47-49. Voir aussi A. RAUWEL, « La fausse charte de Clovis pour Moutiers-Saint-Jean ", Bulletin de la Société des sciences historiques et naturelles de Semur, 113 (2005), p. 66-71.

16. M. DE ChAMBURE, "Inventaire raisonné et descriptif des manuscrits de la Bibliothèque publique de la ville de Semur », 1834, p. 10.

17. L'ouvrage d'A. Vittenet [L'abbaye de Moutier-Saint-Jean (Côte-d'Or), essai historique, préface de J. Toutain, Mâcon, 1938], et toute l'historiographie postérieure sur Moutiers-Saint-Jean, s'appuie fortement sur l'œuvre de Pierre Rouvier.

18. PARIS, BnF, Collection de Bourgogne, 9, f. 123v.

19. Documents conservés à la bibliothèque municipale de Semur-en-Auxois. Nous remercions son conservateur, M. Jean-Claude Sosnowski, de nous avoir autorisé la consultation de ces pièces.

INDEX

Mots-clés : manuscrit

Index géographique : France/Semur-en-Auxois 